\title{
Slow light in quantum dot photonic crystal waveguides
}

Nielsen, Torben Roland; Lavrinenko, Andrei; Mørk, Jesper

Published in:

Applied Physics Letters

Link to article, DOI:

$10.1063 / 1.3103286$

Publication date:

2009

Document Version

Publisher's PDF, also known as Version of record

Link back to DTU Orbit

Citation (APA):

Nielsen, T. R., Lavrinenko, A., \& Mørk, J. (2009). Slow light in quantum dot photonic crystal waveguides. Applied Physics Letters, 94(11), 113111. https://doi.org/10.1063/1.3103286

\section{General rights}

Copyright and moral rights for the publications made accessible in the public portal are retained by the authors and/or other copyright owners and it is a condition of accessing publications that users recognise and abide by the legal requirements associated with these rights.

- Users may download and print one copy of any publication from the public portal for the purpose of private study or research.

- You may not further distribute the material or use it for any profit-making activity or commercial gain

- You may freely distribute the URL identifying the publication in the public portal

If you believe that this document breaches copyright please contact us providing details, and we will remove access to the work immediately and investigate your claim. 


\title{
Slow light in quantum dot photonic crystal waveguides
}

\author{
Torben Roland Nielsen, ${ }^{\text {a) }}$ Andrei Lavrinenko, and Jesper Mørk \\ Department of Photonics Engineering, DTU Fotonik, Technical University of Denmark, Ørsteds Plads 343, \\ DK-2800 Kgs. Lyngby, Denmark
}

(Received 5 February 2009; accepted 27 February 2009; published online 18 March 2009)

\begin{abstract}
A theoretical analysis of pulse propagation in a semiconductor quantum dot photonic crystal waveguide in the regime of electromagnetically induced transparency is presented. The slow light mechanism considered here is based on both material and waveguide dispersion. The group index $n_{g}$ for the combined system is significantly enhanced relative to slow light based on purely material or waveguide dispersion. (C) 2009 American Institute of Physics. [DOI: 10.1063/1.3103286]
\end{abstract}

Since the early experimental reports on slowing down the speed of light beams in atomic vapors, ${ }^{1}$ the study of slow light (SL) phenomena based on actively changing the material dispersion has become a topic of growing interest. Much of this attention is due to potential applications, e.g., as an optical buffer or a phase shifter, applications which are feasible if an efficient SL mechanism can be realized in a compact semiconductor waveguide. ${ }^{2}$ For a typical ridge waveguide (RWG) configuration, SL in quantum dot (QD) structures has theoretically been investigated in the regime of electromagnetically induced transparency (EIT) ${ }^{2,3}$ By applying an externally controlled light source, a variable SL buffer can in principle be realized. Very recently, experimental studies of EIT in QD systems have been performed, ${ }^{4}$ where a coherent absorption dip in a pump-probe experiment has been observed for an optically thin structure. SL based on waveguide dispersion such as in photonic crystal $(\mathrm{PhC})$ waveguides has lately been intensely studied and recent theoretical and experimental studies have shown that group indices higher than 230/40 can be obtained for a narrow/large bandwidth. ${ }^{5-7}$ Since the dispersion properties of slab PhC waveguides are predetermined by the $\mathrm{PhC}$ structure and the bulk material properties, variable slow down factors cannot easily be obtained for fixed frequencies. However, introducing optical nonlinearities into the $\mathrm{PhC}$ are predicted to have significant technological impact on signal processing. ${ }^{8} \mathrm{SL}$ based on QD EIT systems, on the other hand, sets rather strict requirements to the dephasing rates, dipole moments, QD densities, and uniformity of the QD ensemble. In this paper we show, using extensive numerical simulations, that by combining both waveguide and material dispersion the SL effect can be drastically enhanced compared to the individual subsystems. Furthermore, we derive an approximate analytical result that accounts for the scaling behavior of the slow-down factor observed in the full numerical simulations.

We consider a W1 PhC waveguide slab geometry with QDs embedded in the structure (see Fig. 1). The QDs are excited in the ladder arrangement, ${ }^{2,3}$ i.e., the probe beam is resonant with the QD electron-hole ground state transition, while a strong (control) beam is applied between the QD electron ground state and a third excited electron state. The incoming probe is linearly polarized along the $\hat{y}$ axis (TE) and will thus be slowed down due to the $\mathrm{PhC}$ waveguide

\footnotetext{
${ }^{\text {a) }}$ Author to whom correspondence should be addressed. Electronic mail: trol@fotonik.dtu.dk.
}

dispersion and the QD EIT medium. In the following we will just focus on the three QD levels coupled by the optical fields, and neglect any interaction with other energetically close (confined QD or delocalized) states, in which case the QD system can be modeled as a simple three level system. The transition between $|3\rangle$ and $|1\rangle$ is dipole disallowed, while the two remaining transitions are dipole allowed. A full vectorial analysis of the electromagnetic fields is needed in order to give a proper description of pulse propagation in these strongly diffractive $\mathrm{PhC}$ structures. Thus, we use a semiclassical description of the light-matter interaction by combining the finite-difference-time-domain (FDTD) (Ref. 9) and the density matrix methods for studying pulse propagation in a SL QD medium, ${ }^{2}$ thereby treating the electromagnetic fields and the microscopic polarization on an equal footing. ${ }^{10}$ The light-matter interaction is treated within the dipole approximation. When implemented on a FDTD grid, the Liouville equation is solved numerically at every grid point for the QD carrier dynamics without any further approximations. The polarization density which enters Ampere's law is evaluated from the expectation value of the macroscopic polarization via the density matrix. The numerical implementation ${ }^{11}$ follows the method outlined in Ref. 10.

For the numerical results presented in this paper we consider a homogenous InGaAs QD system with parameters similar to Ref. 2. The inhomogeneity of the QD ensemble is neglected for illustration purposes. The electron-hole ground state transition wavelength is $\lambda_{21}=1.38 \mu \mathrm{m}$ while the electron-electron transition wavelength is $\lambda_{32}=12.8 \mu \mathrm{m}$. The corresponding dipole moments are $\mu_{21}=0.68 \mathrm{e} \mathrm{nm}$ and $\mu_{32}=2.46 \mathrm{e} \mathrm{nm}$. For simplicity we use the same dephasing rate $\gamma=80 \mu \mathrm{eV}$ for all QD coherences. ${ }^{12}$ The optical con-
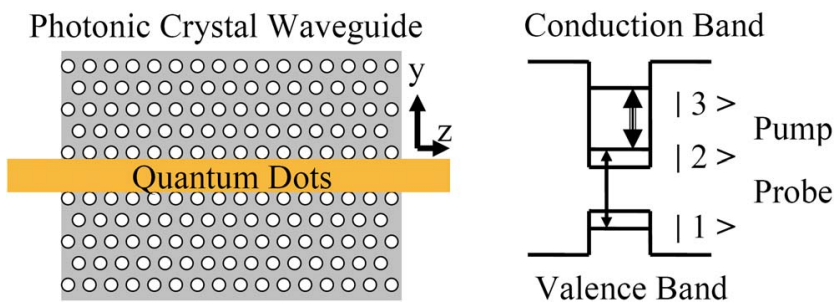

FIG. 1. (Color online) Left: Schematic of the semiconductor QD PhC waveguide system. The QDs are located in the waveguide as indicated by the dark yellow shading. Right: Level scheme of the QDs used for obtaining EIT in a ladder configuration. A weak probe beam is applied between the electron-hole ground state transition $(|2\rangle \leftrightarrow|1\rangle)$, while a strong pump beam is resonant with two electronic states $(|3\rangle \Leftrightarrow|2\rangle)$. 

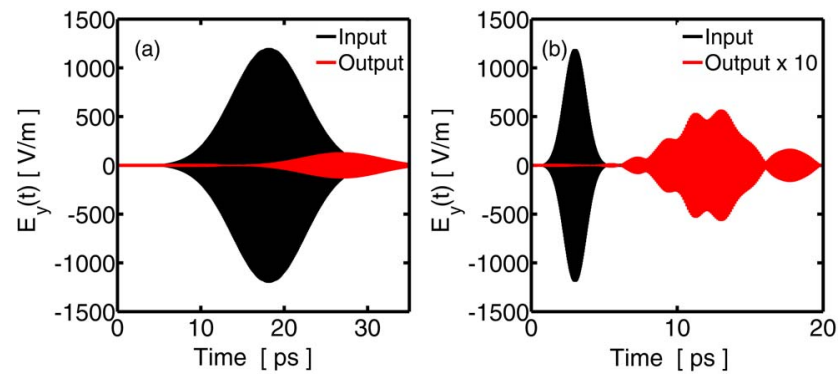

FIG. 2. (Color online) Pulse propagation through a QD PhC waveguide with QDs excited in the ladder configuration. Input (black) and output (red) probe field $E_{y}$ at the waveguide midpoint as a function of time. The pump power density is $I_{P}=0.8 \mathrm{MW} / \mathrm{cm}^{2}$ and the incoming probe beam has a FWHM of $10 \mathrm{ps}$ (a) and $1.66 \mathrm{ps}$ (b).

finement factor reads $\Gamma=14.6 \times 10^{-3}$, which corresponds to ten QD layers in a $\mathrm{PhC}$ WG membrane structure with an average sheet density of $9.6 \times 10^{10} \mathrm{~cm}^{-2}$. The PhC WG structure is similar to the one considered in Ref. 5, only scaled down to support a SL mode at the electron-hole ground state transition wavelength. This leads to a lattice pitch $\Lambda=292 \mathrm{~nm}$ and a hole diameter $D=0.6 \Lambda$, and the total $\mathrm{PhC}$ waveguide length is approximately $22 \mu \mathrm{m}(75$ periods). The refractive background index reads $n_{b}=3.5$.

To study the group index $n_{g}$ for the combined system based on both material and waveguide dispersion, we compare three different scenarios: (1) Pulse propagation in a passive PhC W1 waveguide structure, (2) a RWG with QDs excited in the ladder configuration, and (3) final pulse propagation in the active QD $\mathrm{PhC} \mathrm{W} 1$ waveguide structure. Throughout, we describe the QD ensemble as an effective medium smeared out over the waveguide region, ${ }^{2,3}$ thus implying the same QD properties at each FDTD lattice site. The three level ladder QD system depicted in Fig. 1 is driven with the probe beam which is resonant with electron-hole ground state transition and polarized along the $\hat{y}$ axis. The continuous wave control beam is resonant with the electronelectron transition, polarized along the $\hat{z}$ axis and propagates in the direction perpendicular to the $\mathrm{PhC}$ membrane. Throughout the simulations presented here, we assume that the control beam Rabi energy $\hbar \Omega_{C}=\mu E_{C} / 2$ is much larger than the probe Rabi energy $\hbar \Omega_{P}=\mu E_{P} / 2$, although this is not a limitation of the numerical scheme. The incoming probe beam has a temporal Gaussian envelope with full width half maximum (FWHM) $\tau_{p}$ and a peak amplitude $E_{P}$. For the numerical results related to the group index, we consider probe pulses whose spectral width is well located within the two EIT absorption peaks, which arise due to the dressing of the system by the control beam, such that $\tau_{p} \geq 10 / \Omega_{C}$. The group index is then determined by a time of flight method ${ }^{5}$ giving the delay time between the peak of the pulse just before the entrance and just after the exit of the PhC WG. In order to remove the effects of reflection from the $\mathrm{PhC}$ waveguide, the pulse peak time at the entrance is determined by first performing a simulation with a simple straight waveguide without any $\mathrm{PhC}$ present. Figure 2(a) shows such a typical example of the $E_{y}$ field component at the input and output ports for a pulse propagating through the QD $\mathrm{PhC}$ waveguide. The pulse is seen to propagate fairly undistorted through the waveguide, with only a small temporal widening of the pulse. Thus in this regime a time delay between the inand output pulses can be attributed and hence a group index

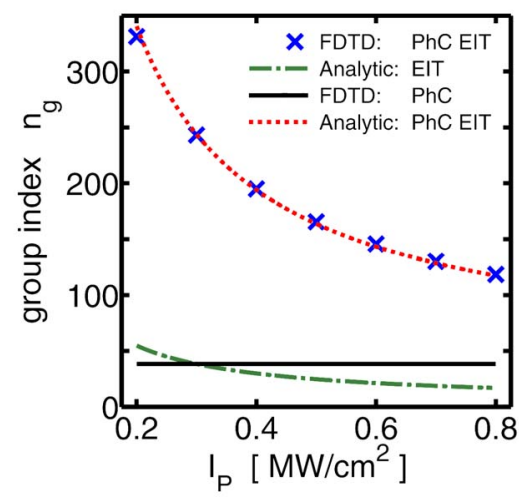

FIG. 3. (Color online) Group index as a function of pump power $I_{P}$ for three different scenarios corresponding to the $\mathrm{PhC}$ waveguide with QDs (blue crosses), analytic RWG with QDs (green dashed dotted line), and the passive $\mathrm{PhC}$ waveguide (black line). The red dotted curve is given by Eq. (1) with $n_{g}=38.5$ and $E_{d}=0.535$. The QDs are excited in the ladder EIT configuration. The incoming probe beam has a temporal FWHM $\tau_{p}=10$ ps.

can be extracted. Furthermore, a drop in the transmittance is also observed. The actual total delay and the reduction of the transmittance depend on the length of the $\mathrm{PhC}$ waveguide, giving larger delay and lower transmittance for longer waveguides. Moreover, compared to the RWG EIT system we observed an enhanced drop in the transmission. A detailed analysis of the absorption properties is however beyond the scope of this letter, but a similar effect has recently been reported in Ref. 13. Short probe pulses on the other hand, will in general, experience strong dispersion and the probe pulse is broadened or even broken up. ${ }^{6,14,15}$ In this regime the dynamic becomes nonlinear and a clear identification of, e.g., slow-down factors becomes impossible [see Fig. 2(b)].

In Fig. 3 we plot the group index for pulses propagating through a passive $\mathrm{PhC}$ waveguide, $\mathrm{RWG}$ embedded with QDs, and the QD PhC waveguide system as a function of the applied pump power density of the control beam $I_{P}$ $=c \varepsilon_{0} n_{\mathrm{bac}}\left|E_{C}\right|^{2}$. The probe pulse duration is fixed to $\tau_{p}$ $=10 \mathrm{ps}$. The passive $\mathrm{PhC}$ waveguide group index is of course independent of the pump power density. The group index for the RWG EIT system is determined from the analytic model ${ }^{2}$ and decreases with increasing pump power density and reaches a plateau equal to the back ground index $n_{b}$ at high densities. As the pump power density is lowered the group index increases dramatically, as also discussed in Refs. 2. The group index for the combined system, which explores both material and waveguide dispersion, increases as the pump power is decreased, thus showing the same dependency as the simple RWG EIT system. It is observed, however, that the group index is increased dramatically for the combined system compared to the individual subsystems. Notice that a small change in the pump power density gives a large change in group index for the combined system compared to the RWG EIT system. Thus, by combining both material and waveguide dispersion it is possible to enhance the group index as well as obtaining a variable and controllable group index in an ultrasmall waveguide structure.

It is a complex task to determine the group index analytically in such systems as in general the material dispersion will influence the waveguide dispersion properties. However, the group velocity in a $\mathrm{PhC}$ waveguide structure can be estimated as follows for an ideal infinitely extended system 
with linear constitutive material relations. The harmonic magnetic eigenmodes are the solutions to the equation $\omega^{2} / c^{2}=\langle\mathbf{H}, \boldsymbol{\Theta} \mathbf{H}\rangle /\langle\mathbf{H}, \mathbf{H}\rangle$ where $\boldsymbol{\Theta}=\nabla \times \varepsilon(\mathbf{r}, \omega)^{-1} \nabla \times$. Solving this equation must be performed in a self-consistent way if the dielectric function $\varepsilon$ is frequency dependent. For a propagating mode with propagation constant $\beta$ the group velocity is then evaluated as $v_{g}=d \omega / d \beta$. The total derivative of the eigenvalue $\omega$ with respect to $\beta$ may be evaluated as ${ }^{16}$

$$
\frac{2 \omega}{c^{2}} v_{g}=\frac{d}{d \beta} \frac{\langle\mathbf{H}, \boldsymbol{\Theta} \mathbf{H}\rangle}{\langle\mathbf{H}, \mathbf{H}\rangle}=\frac{\partial}{\partial \beta} \frac{\langle\mathbf{H}, \boldsymbol{\Theta H}\rangle}{\langle\mathbf{H}, \mathbf{H}\rangle}+v_{g} \frac{\partial \varepsilon}{\partial \omega} \frac{\partial}{\partial \varepsilon} \frac{\langle\mathbf{H}, \boldsymbol{\Theta} \mathbf{H}\rangle}{\langle\mathbf{H}, \mathbf{H}\rangle} .
$$

For low-loss dielectrics, with negligible imaginary dielectric function, the group velocity then reads ${ }^{16} 2 v_{g}=2 v_{g}^{\mathrm{PhC}}$ $-\omega E_{d}[\partial \ln (\varepsilon) / \partial \omega] v_{g}$, where $v_{g}^{\mathrm{PhC}}$ is group velocity for the $\mathrm{PhC}$ waveguide without any material dispersion, and $E_{d}$ $=\langle\mathbf{D}, \mathbf{E}\rangle_{\mathrm{QD}} /\langle\mathbf{D}, \mathbf{E}\rangle$ is the fraction of the electric energy inside the dielectric having a frequency dependent dispersion. Using $\varepsilon=n^{2}$, the partial derivative of $\ln (\varepsilon)$ may be expressed in terms of the dispersive group index, and the total group index can be written as

$$
n_{g}=n_{g}^{\mathrm{PhC}}\left[1+E_{d}\left(\frac{n_{g}^{\mathrm{EIT}}}{n_{b}}-1\right)\right] \text {. }
$$

This result shows that the group index has a contribution that scales linearly with the product of $n_{g}^{\mathrm{PhC}}$ and $n_{g}^{\mathrm{EIT}}$. The total group index in a system which combines both material and waveguide dispersion may then be evaluated from the passive $\mathrm{PhC}$ waveguide group index $n_{g}^{\mathrm{PhC}}$, filling factor $E_{d}$, and from the QD ensemble group index $n_{g}^{\text {EIT }}$ alone. In Fig. 3 we have also plotted the prediction of Eq. (1). The electric energy in side the QD region is evaluated from the numerical simulations as follows: $E_{d}=\int d t\langle\mathbf{D}, \mathbf{E}\rangle_{\mathrm{QD}} / \int d t\langle\mathbf{D}, \mathbf{E}\rangle$. From our calculations we obtain $E_{d}=0.535$ and $n_{g}^{\mathrm{PhC}}=38.5$. We observed good agreement between numerical simulations and theory.

In conclusion, we have investigated light slow-down in a $\mathrm{PhC}$ structure including QDs. The slow-down factor may be significantly enhanced compared to systems relying only on material or waveguide dispersion. This could be important in achieving efficient and variable control over pulse propagation in compact semiconductor waveguides.

This work has been partially financed by the European Commission through the IST Project No. 29283, QPhoton, the project QUEST financed by the Danish Research Councils, and the project NATEC financed by the VILLUM KANN RASMUSSEN FOUNDATION. The authors also acknowledge access to the Danish Center for Scientific Computing (DCSC) at the Technical University of Denmark under Grant No. DCSC CPU-0107-12.

${ }^{1}$ L. V. Hau, S. E. Harris, Z. Dutton, and C. H. Behroozi, Nature (London) 397, 594 (1999).

${ }^{2}$ C. J. Chang-Hasnain, P. C. Ku, J. Kim, and S. H. Chuang, Proc. IEEE 91, 1884 (2003).

${ }^{3}$ P. Kaer Nielsen, H. Thyrrestrup, J. Mørk, and B. Tromborg, Opt. Express 15, 6396 (2007).

${ }^{4}$ S. Marcinkevičius, A. Gushterov, and J. P. Reithmaier, Appl. Phys. Lett. 92, 041113 (2008).

${ }^{5}$ R. S. Jacobsen, A. V. Lavrinenko, L. H. Frandsen, C. Peucheret, B. Zsigri, G. Moulin, J. F. Pedersen, and P. I. Borel, Opt. Express 13, 7861 (2005)

${ }^{6}$ L. H. Frandsen, A. V. Lavrinenko, J. F. Pedersen, and P. I. Borel, Opt. Express 14, 9444 (2006).

${ }^{7}$ J. Li, T. P. White, L. O'Faolain, A. Gomez-Iglesias, and T. F. Krauss, Opt. Express 16, 6277 (2008).

${ }^{8}$ M. Soljacic and J. D. Joannopoulos, Nature Mater. 3, 211 (2004).

${ }^{9}$ A. Taflove and S. C. Hagness, Computational Electrodynamics (Artech House, Boston, 2005).

${ }^{10}$ R. W. Ziolkowski, J. M. Arnold, and D. M. Gogny, Phys. Rev. A 52, 3082 (1995).

${ }^{11}$ Our numerical implementation is equivalent to Eqs. (1)-(4b) of Ref. 10 with the obvious changes needed in describing a semiconductor system. Notice that the Eq. (4a) of Ref. 10 is the equivalent of Eq. (1) in Ref. 2.

${ }^{12}$ P. Borri, S. Schneider, W. Langbein, and J. Dieter Bimberg, J. Opt. A, Pure Appl. Opt. 8, S33 (2006).

${ }^{13}$ N. A. Mortensen and S. Xiao, Appl. Phys. Lett. 90, 141108 (2007).

${ }^{14}$ R. W. Boyd, D. J. Gauthier, and A. L. Gaeta, Phys. Rev. A 71, 023801 (2005).

${ }^{15}$ P. Jänes, J. Tidström, and L. Thylén, J. Lightwave Technol. 23, 3893 (2005).

${ }^{16}$ J. Lægsgaard, A. Bjarklev, and S. E. B. Libori, J. Opt. Soc. Am. B 20, 443 (2003) 